\title{
Editorial
}

\section{Clinical and Diagnostic Pathology: Inaugural Message from the Editor-in-Chief}

\section{Rawhi Omar* \\ Pathology and Lab Med, University of Louisville Medical School, USA}

Increasingly, physicians are relying more than ever on the clinical laboratory to provide them with diagnostic information, whether be it partially diagnostic as in Clinical Pathology (blood and body fluid cellularity and biochemistry) or definitive as in Anatomic (biopsy) Pathology. On both fronts, along with contributory or basic diagnostic information, it is also expected that pathologists are able to deliver such information, and perhaps more, from analyzing an increasingly smaller sample that can be procured via a procedure that is minimally invasive (such as small or fine needle aspiration) or completely non-invasive (exfoliative cytology, urine, saliva etc).

These dynamics are most-illustrative in the field of cancer diagnosis and treatment. Almost gone are the days when the diagnostic pathologist's work could be completed (diagnosis rendered) solely by a microscopic review of a few routinely (H\&E) stained microscopic slides. Not only the sample now is much smaller, but in addition to the basic anatomic pathology diagnosis of malignancy, you are expected to also provide new tumor profiles: an immunophenotypic (antigenic characterization using immunohistochemical methods) and molecular (selected genetic and epigenetic characterization using molecular biology methods). These profiles now are at the core of the so-called "personalized" medicine in oncology as they can be used to build a specific prognosis and tailor a specific chemotherapeutic regimen (e.g., thyrosine kinase Inhibitors) to a specific cancer patient.

Moving cancer diagnosis (accurate, yet detailed and personalized) into the minimally invasive or non-invasive procedural category means getting more out of less. Accordingly, the search is on for more specific tumor markers or cancer indicators and especially those that can be identified from few DNA or cellular fragments floating in a standard blood sample: the so-called "liquid biopsies". While currently there is no single marker or single molecule that can be claimed as a definitive diagnostic marker of a specific cancer type, this frontier is being vigorously explored. One promising example is that of the socalled "ENOX2 proteins". These are novel markers claimed to be cancer type specific (Ecto-Nicotineamide Oxidase type enzymes translated only in embryonic and cancer cells as opposed to ENOX1 which are a similar class but expressed on normal adult cells). The hope is that such markers can be identified on cells or cell fragments contained in "liquid biopsies", making the combination uniquely diagnostic. Future research activities in Pathology (disease associated tissue changes) may not stop at the molecular level but are, indeed, trending towards even smaller structural units such as atoms or even subatomic particles (nanopathology?).

The described realities and challenges are keeping pathologists on their toes, not only to look for newer diagnostic targets or testing methods, but also to keep up with the discoveries of peers and affiliated scientists. It is to facilitate the latter, i.e., exchange of new and reliable (peer-reviewed) information, that the journal of "Clinical and Diagnostic Pathology" is being inaugurated. It will be an open forum for contributors of relevant information from around the World: original research data, reviews, opinions etc.. Readers everywhere will have a free and open Internet access to this information.

With those thoughts in mind, I invite you to submit your academic work with the promise for an expedient and fair editorial and peer review process, so that worthy information can be published and shared in a timely manner.

On behalf of the Editorial Board and publishing team, welcome aboard the new journal of "Clinical and Diagnostic Pathology".
Copyright: (C2016 Omar R. This is an open-access article distributed under the terms of the Creative Commons Attribution License, which permits unrestricted use, distribution, and reproduction in any medium, provided the original author and source are credited.
Correspondence to: Rawhi Omar, Professor of Pathology and Lab Med, University of Louisville Medical School, USA; E-mail: raomar12@gmail.com

Received: August 10, 2016; Accepted: August 23, 2016; Published: August 26, 2016 